IdeAs

Idées d'Amériques

$7 \mid 2016$

Cinéma et histoire dans les Amériques

\title{
La « reconquête » des favelas et les J.O. de Rio
}

\section{Hervé Théry}

\section{OpenEdition}

Journals

Édition électronique

URL : https://journals.openedition.org/ideas/1407

DOI : 10.4000/ideas. 1407

ISSN : 1950-5701

\section{Éditeur}

Institut des Amériques

\section{Référence électronique}

Hervé Théry, «La « reconquête » des favelas et les J.O. de Rio », IdeAs [En ligne], 7| 2016, mis en ligne le 27 juin 2016, consulté le 18 octobre 2022. URL : http://journals.openedition.org/ideas/1407 ; DOI : https://doi.org/10.4000/ideas. 1407

Ce document a été généré automatiquement le 18 octobre 2022

\section{(c) (1) (9)}

Creative Commons - Attribution - Pas d'Utilisation Commerciale - Pas de Modification 4.0 International - CC BY-NC-ND 4.0

https://creativecommons.org/licenses/by-nc-nd/4.0/ 


\title{
La « reconquête » des favelas et les J.O. de Rio
}

\author{
Hervé Théry
}

\section{Introduction}

1 Depuis des années, les pouvoirs publics avaient pratiquement renoncé à exercer leur autorité dans les favelas de Rio et à y assurer les services que l'on pourrait attendre de la deuxième ville du Brésil, la vitrine offerte au monde, notamment aux touristes qui y affluent et, cette année, l'accueil des Jeux Olympiques. Le narcotrafic y avait si bien prospéré que c'étaient de fait ses chefs qui y faisaient régner l'ordre nécessaire à la bonne marche de leur commerce de drogues, "protégeaient " les commerçants en échange d'une contribution financière, et tenaient leurs habitants par un mélange de menace et de distribution de petits services, pour s'assurer leur bienveillance ou au moins leur silence. Pourquoi en ont-ils entrepris la « reconquête »?

\section{Les favelas et la ville}

Cest que les favelas représentent une part croissante de la population de la ville. En vingt ans, entre 1991 et 2010, la populationde cesbidonvillesest passée d'un peu moins de 900000 àprès d'un million et demi d'habitants, uneaugmentationde plus de $65 \%$. Les favelas du sud et du centre de la ville, enclavées dans des zones urbaines consolidées, ne peuvent plus s'étendre, mais leur population continue à croître, par la construction de nouveaux étages au-dessus des logements précédents, non sans risque puisque les maisons, construites sans architecte sur des terrains en pente, ne supportent pas toujours ces surcharges. Dans la zone ouest de Rio, elles se sont en revanche beaucoup étendues en conquérant de nouveaux espaces, ce qui a eu de forts impacts sur l'environnement, notamment dans le parc national de Tijuca, la plus grande forêt urbaine au monde. 
3 Au total, les favelas ont ainsi vu leur part dans la population de la ville (6,5 millionsd'habitants, 12 millions dans l'agglomération urbaine au recensement de 2010, le dernier en date) passer d'un peu moins de $10 \%$ en 1960 à $22 \%$ en 2010. La population des favelas progresse beaucoup plus vite que celle des quartiers de l'« asphalte ", pour reprendre la façon dont les habitants appellent le reste de la ville.

Population des favelas par AP (zones de planification) 1991-2010

\begin{tabular}{|l|l|l|l|l|l|l|}
\hline & 1991 & $\mathbf{2 0 0 0}$ & $\mathbf{2 0 1 0}$ & \% & \multicolumn{2}{l|}{ Variation 2000-2010 } \\
\hline & & & & en favela & en favelas & hors favelas \\
\hline Total & 882483 & 1092476 & 1443773 & 23 & 19 & 5 \\
\hline AP 1 - Centro & 85182 & 76787 & 28060 & 27 & 28 & 4 \\
\hline AP 2 - Zona Sul & 127104 & 146538 & 58305 & 33 & 15 & -1 \\
\hline AP 3 - Zona Norte & 480524 & 544737 & 149014 & 23 & 11 & -1 \\
\hline AP 4 - Barra/Jacarepaguá & 72182 & 144394 & 13310 & 6 & 53 & 28 \\
\hline AP 5 - Zona Oeste & 117491 & 180020 & 34369 & 13 & 15 & 8 \\
\hline
\end{tabular}

Source : Estimation IPP à partir du recensement IBGE de 2010

Les six plus grandes favelas en 2010

\begin{tabular}{|l|l|l|l|}
\hline Favelas & AP* & Domiciles & Population \\
\hline Rocinha & 2 & 23347 & 69156 \\
\hline Maré & 3 & 20897 & 64056 \\
\hline Rio das Pedras & 4 & 22131 & 63453 \\
\hline Alemão & 3 & 18226 & 60555 \\
\hline FazendaCoqueiro & 5 & 14266 & 45366 \\
\hline Vila Cruzeiro & 3 & 9791 & 35971 \\
\hline
\end{tabular}

Source : Estimation IPP à partir du recensement IBGE de 2010 *Aire de planification.

4 Parmi les nouvelles favelas, un tiers se sont installés dans les régions de Barra da Tijuca et Jacarepaguá, proches des sites olympiques. La croissance y a été rapide, avec des taux de $7,5 \%$ par an à Jacarepaguá et de près de $10 \%$ à Barra da Tijuca, presque six fois plus vite qu'au cours de la période précédente. À ce rythme, la majorité de la population de Jacarepaguá vivra dans les favelasen 2024. 


\section{Les premières grandes opérations}

5 Cette situation a été une des raisons majeures du lancement d'une opération de «reconquête» des favelas de Rio de Janeiro. Ce mot a été utilisé pour caractériser ce qu'a connu la ville dans les derniers jours de novembre 2010, de véritables scènes de guerre entre les forces de sécurité brésiliennes et des centaines de trafiquants de drogue retranchés dans le complexe de favelas de la Vila Cruzeiro et du Complexo do Alemão. Les premières ont lancé un véritable assaut dans ce groupe de bidonvilles, au cours duquel au moins quarante personnes ont été tuées. La presse suivant de très près les troupes de choc, des images spectaculaires ont été aussitôt publiées, comme, par exemple, celles que l'on peut encore voir sur le site du Boston Globe, The Big Picture ${ }^{1}$.Le journal O Dia du 29 novembre 2011 racontait: "La police brésilienne a hissé le 28 novembre, en signe de victoire, le drapeau national au sommet d'un bastion de narcotrafiquants situé dans le nord de Rio. En l'espace de deux heures, quelque 2600 hommes, parachutistes et troupes de choc de la police, appuyés par des blindés et des hélicoptères, ont pris le contrôle du Complexo do Alemão, un ensemble de quinze favelas dans lequel vivent 400000 personnes » et conclut: "Il s'agit d'une journée historique pour les honnêtes citoyens de Rio."

6 Peu après, les télévisions et les quotidiens, les grands hebdomadaires brésiliens et étrangers ont publié des reportages-choc, comme L'Express, qui titrait «Scènes de guerre à Rio : la police reprend une favela avec des blindés ", Le Point "Les blindés investissent les favelas à Rio de Janeiro ». Paris-Match, sous le titre "De violents combats entre trafiquants et policiers dans les favelas de Rio de Janeiro ont fait un mort et une dizaine de blessés » écrivait : «Le calme est semble-t-il revenu dans les favelas du nord de Rio. Le renfort de 4500 hommes n'aura pas été de trop pour maîtriser une situation explosive [...] La municipalité, qui accueillera successivement le Mondial de football en 2014 et les Jeux olympiques d'été en 2016, a tenu à rassurer sur sa capacité à contrôler les violences afin d'assurer la sécurité de ces deux évènements planétaires. »Et le journaliste cite José Beltrame, secrétaire à la Sécurité de l'État de Rio: "“C'est un problème d'une région, dans un quartier bien précis de la ville. Ce n'est pas Rio de Janeiro" "

\section{Une occupation en trompe-l'œil ?}

7 Après l'occupation de Vila Cruzeiro, et du Complexo do Alemão, le ministre de la Défense, NelsonJobim, avait déclaré: "Cela montre que le Brésil a le sens de la responsabilité et qu'il est capable de résoudre ses problèmes avant de recevoir de grands événements». En réalité, la police et les militaires étaient entrés dans le Complexo do Alemão sans grande résistance. Pour les narcotrafiquants, tenter de résister vraiment aurait été un suicide, faisant de nombreux morts parmi les bandits, les militaires mais aussi les résidents pris dans les échanges des tirs. Des tonnes de drogue ont été saisies, des trafiquants ont été arrêtés, des armes légères confisquées, mais peu de chose par rapport à l'arsenal des bandits, qui avaient même des mitrailleuses anti-aériennes, comme s'en sont rendus compte plusieurs fois les hélicoptères de la police.

8 Selon un observateur bien informé, Reinaldo Azevedo, «depuis le début du siège, les forces de sécurité négociaient avec le banditisme. Joseph Jr., de l'ONG AfroReggae, a été 
l'une des personnes qui ont fait le pont. L'occupation n'a été décidée qu'après qu'un accord ait été conclu, il a été établi que les forces de sécurité pourraient "envahir" la zone sans résistance. Les bandits ont accepté de ne pas trop résister et l'État leur a donné le droit de s'échapper ».

9 Cette reconquête était évidemment indispensable et devra se poursuivre dans d'autres favelas de Rio, trop longtemps abandonnées par les pouvoirs locaux. Elle était attendue depuis au moins vingt ans par des habitants otages du trafic de drogue qui ne supportaient plus de voir tous les actes de leur vie quotidienne régis par les trafiquants : les commerçants ont été plusieurs fois sommés de fermer leurs magasins quand un chef de gang était tué dans un règlement de compte, le trafic a progressivement accaparé la distribution du gaz, de l'eau, de l'électricité et l'accès piraté - à Internet et à la télévision numérique.

Mais une fois cette réoccupation acquise, il aurait fallu faire un travail de police approfondi pour arrêter les trafiquants, ce qui n'a pas été fait jusqu'à présent dans les treize bidonvilles pacifiés lors des précédentes opérations. Toujours selon Reinaldo Azevedo, «dans onze d'entre eux, le trafic fonctionne normalement. La logistique a changé, ainsi que le comportement des trafiquants de drogue, des droits minimums sont garantis par la police, mais le commerce de drogues est resté inchangé. Les soldats des trafiquants deviennent inutiles dans les bidonvilles où les UPP (Unités de police de pacification) sont arrivées, ils ont déménagé vers les bidonvilles où les policiers ne sont pas encore présents ».

11 Au-delà de cette opération spectaculaire - et conçue pour l'être -, que peut-on attendre de l'avenir ? La reconquête des favelas par les pouvoirs publics va-t-elle se poursuivre?

Elle s'est déjà prolongée en 2011, où la cible a été la favela de Rocinha, la plus peuplée de Rio. Elle comptait en 2010, selon les données du Recensement, 68530 habitants, et sa population y a augmenté de $23 \%$ en dix ans. Sa reconquête a commencé le dimanche 13 novembre 2011, menée par la Police militaire de l'État de Rio de Janeiro (PM) et les fusiliers marins. Un an plus tard une Unité de police pacificatrice (UPP) permanente y a été inaugurée avec 700 policiers communautaires spécialement formés, la $28^{\mathrm{e}}$ unité installée dans l'une des 750 favelas de Rio. Comme on ne peut circuler en voiture que dans $20 \%$ de la favela, les policiers patrouillent en moto et à pied dans les quartiers, aidés par cent caméras de surveillance. "La police est ici et va y rester pour toujours ", a promis le gouverneur de Rio lors d'une cérémonie tenue au pied de la favela, sous une passerelle blanche conçue par l'architecte de Brasília, Oscar Niemeyer.

De nouvelles opérations ont été menées au début du mois d'octobre 2013 dans huit favelas du Complexo do Lins, dans la proche zone nord (proche du quartier de Méier). Puis le dimanche 30 mars 2014, la Police Militaire a occupé, toujours dans la Zone Nord, les bidonvilles du "complexe de Maré », qui compte dans ses quinze favelas un peu moins de 130000 habitants, pour y installer la $39^{\mathrm{e}}$ UPP de Rio. La région, une des plus violentes de la capitale, est considérée comme stratégique car située entre la LinhaVermelha, la LinhaAmarela et l'Avenida Brasil - les principales artères de la ville - et l'aéroport international Tom Jobim (Galeão). Il n'y a eu aucun affrontement lors de l'occupation, 102 personnes avaient été détenues depuis le 22 mars, quand avaient commencé les préparatifs de l'opération.

14 Une fois encore, les drapeaux du Brésil et de l'État de Rio de Janeiro ont été déployés, symbolisant la reprise du territoire par l'État. Le Secrétaire à la sécurité publique, José Mariano Beltrame, a annoncé le 28 avril 2015 que les prochaines favelas occupées 
seraient Pedreira e Chapadão, Lagartixa et Quitungo, proches du ComplexoEsportivo de Deodoro où auront lieu onze épreuves olympiques des jeux de 2016 et quatre épreuves para-olympiques.

\section{Une stratégie sélective}

Si l'on reporte les informationssur la carte, on perçoit en effet une claire stratégie territoriale. Les premières UPP ont été créées dans la zone de planification 1 (AP1), aux abords du centre, et dans la zone 2, celle qui couvre la zone sud, riche et touristique. Elles ont été disposées de façon à couvrir chacune une ou plusieurs des petites favelas qui se nichent sur les morros, les mornes tropicaux qui hérissent cette région de relief tourmenté.

\section{La reconquête des favelas de Rio de Janeiro}

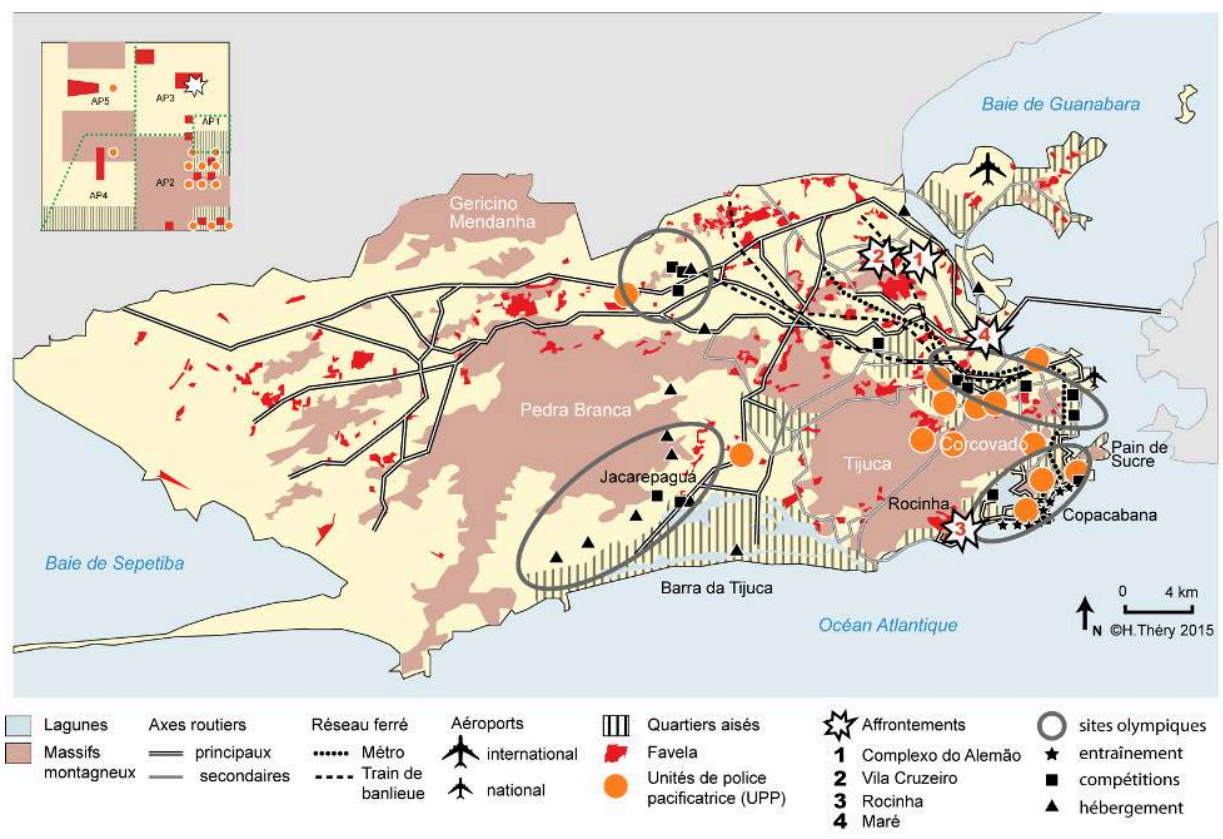

La conquête de la Vila Cruzeiro et du Complexo do Alemão inaugure une nouvelle phase, celle de la reconquête des grands ensembles de favelas de la zone nord, plus plane et plus pauvre, mais d'où l'on accède facilement au centre et qui menace la route vers l'aéroport. Après quoi on a repris le contrôle de la Rocinha, aux confins de la zone 4 ; il restera à finir la récupération de celle-ci, où la croissance rapide des favelas fait de l'ombre aux nouveaux quartiers chics de la Barra da Tijuca. La zone 5, pauvre et lointaine, pourra attendre, l'essentiel sera fait, dégager les accés aux installations olympiqueset éviter que des incidents ne gâchent le bon déroulement des Jeux. 


\section{BIBLIOGRAPHIE}

Beyer, Antoine, «L'échéance des Jeux Olympiques de 2016 et les stratégies de restructuration du transport métropolitain de Rio de Janeiro ", Confins 12 | 2011, URL : http://confins.revues.org/ 7087 ; DOI : 10.4000/confins.7087

Chetry, Michael, «L'insertion des habitants des favelas de Rio de Janeiro dans la ville : le cas des pratiques de consommation et de loisirs ", Confins [13 | 2011, URL : http://confins.revues.org/ 7244 ; DOI : 10.4000/confins.7244

Théry, Hervé, Le Brésil, pays émergé, collection Perspectives géopolitiques, Armand Colin, ISBN : 9782200278281, 2014, 304 p.

\section{NOTES}

1. Voir : http://archive.boston.com/bigpicture/2010/11/rios_drug_war.html

\section{AUTEUR}

\section{HERVÉ THÉRY}

Directeur de recherche émérite au CNRS, Professor visitante na Universidade de Sao Paulo (USP), hthery@aol.com 